\title{
Service Managers' Experiences of how the Participation of People with Intellectual Disabilities can be Promoted in Swedish Group Homes
}

Original article

Leena Berlin Hallrup ${ }^{1}$, Christine Kumlien ${ }^{12}$ and Elisabeth Carlson ${ }^{1}$

${ }^{1}$ Department of Care Science, Faculty of Health and Society, Malmö University

2Department of Cardio-Thoracic and Vascular Surgery, Skåne University Hospital, Sweden

Running title

Service Managers' Experiences of Participation

\section{Correspondence to:}

Leena Berlin Hallrup

Department of Care Science, Faculty of Health and Society

Malmö University

Jan Waldenströms gata 25

20506 Malmö

SWEDEN

E-mail: leena.berlinhallrup@gmail.com

Phone: +46 705531800 


\section{Abstract}

Background People with intellectual disabilities in staffed group homes often need lifelong support and dependency on others. Thereby, special demands are placed on staff and service managers to ensure opportunities for participation in everyday life. This study aims to explore how service managers promote participation in Swedish group homes for adults with intellectual disabilities.

Method A qualitative research design involving individual interviews with 14 service managers was used to gain an understanding of how the participation of adults with intellectual disabilities can be promoted in Swedish group homes.

Findings The findings comprise two main themes; Creating preconditions for participation and Barriers for promotion of participation.

Conclusions Service managers experienced that promoting service user participation in group homes was an important part of their responsibility. The findings indicate that structural strategies such as coaching, supervision and reflection are important and should be further developed.

Keywords Experiences, group home, participation, people with intellectual disabilities, service manager 


\section{Introduction}

The ideological disability policy emphasizes that people with intellectual disabilities (ID) should be given the opportunity to participate in society (United Nations, 2006) and also when residing in group homes (The Swedish Agency for Participation, 2015; The National Board of Health and Welfare, 2017). Participation is defined as a person's involvement in a life situation (World Health Organization, 2001). The definition of participation in the present study originates from active support, described as the relationship between staff and the people they support that provides assistance to enable a person to successfully participate in meaningful activities and social relationships (Bigby, Bould, \& Beadle-Brown, 2017; Mansell \& BeadleBrown, 2012). A recent study has also shown that before participation can be created, a trustful relationship must be established between the professionals and people with ID (Collings, Dew \& Dowse, 2018). However, in what way adults with ID can be involved and participate in everyday life, when residing in group homes, may also depend on how that support is organized by service managers. Service managers are responsible for developing and promoting the support provided by frontline staff (Deveau \& McGill, 2016). The current study focuses upon the experiences of service managers who are directly responsible for managing and supporting staff in group homes for adults with ID.

People with ID in staffed group homes often need lifelong support and are inadvertently put in a position of dependency on others (Bailey, Doody, \& Lyons, 2016). Thereby, special demands are placed on staff, service managers and the organization to ensure that people with ID have the opportunity to participate in everyday life. Previous studies have revealed that support to staff from their immediate manager may be an important factor for development and improvement of neccesary staff skills (Deveau \& McGill, 2016). Studies have also shown that managers' leadership and way of motivating staff, can result in better outcomes for residents in group homes in terms of participation and increased independence (Bigby, Knox, Beadle- 
Brown, \& Clement, 2015; Deaveau \& McGill, 2014; Jackson, 2011). Beadle-Brown et al., (2014) state that practice leadership provided by the service manager is of importance when supporting staff to pay close attention to the provision of active support. The concept of practice leadership is defined as the development and maintenance of good staff support for residents, for example by service managers observing how staff members work and act as well as providing immediate feedback and supervision in everyday practice (Deveau \& McGill, 2016; Mansell \& Beadle-Brown, 2012). Moreover, practice leadership also involves staff working in accordance with the active support evidence based model, which facilitates autonomy in daily life for people with ID (Beadle-Brown et al., 2014; Deveau \& McGill, 2014; Mansell \& BeadleBrown, 2012). This combination of practice leadership and active support provides enhanced support for staff as well as a better outcome for people with ID (Beadle-Brown et al., 2014; Mansell \& Beadle-Brown, 2012). Bigby and Beadle-Brown (2016 a) also noted that the challenges facing service managers not only concerns how to provide the necessary skills for working according to the active support model but also how to motivate staff members to employ the model in a consistent and systematic way to promote participation.

The evidence based models such as active support and practice leadership are not yet implemented in Sweden (The National Board of Health and Welfare, 2017). However, a nonevidence based model which is used in some organizations is the participation model. The participation model aims to provide opportunities for service users to participate in formally organized activities in group homes. The model is based on dialouge discussions, conducted in smaller groups of residents led by a supervisor. The dialouge discussion is evaluated by the service manager in order to enable residents' opinions and interests to be taken serious and implemented in the everyday practice (Gullacksen \& Hejdedal, 2014). Further, the model of participation is a framework that can support service managers when creating an organizational structure that promotes opportunities for participation and social inclusion at a micro level such 
as a group home. Consequently, the model of participation may be an important part of organizational strategies to be used by the service manager to achieve and ensure participation, rather than being synonymous to practice leadership. Moreover, the participation model by Gullacksen and Hejdedal (2014) is not empirically tested in research but can be seen as a first step in strenghtening empowerment for service users in order to facilitate participation in social activities in a broader community in the society as described by Bigby, Andersson, and Cameron (2017).

However, previous research shows, that barriers for participation in everyday life for people with ID residing in group homes, still exist. Such barriers may occur due to the approach and attitudes of staff (Bigby, Knox, Beadle-Brown, Clement, \& Mansell, 2012; Deveau \& McGill, 2014) and to the delivery of support and care (Bigby, Frawley, \& Ramcharan, 2014; Mansell \& Beadle-Brown, 2012). Further aspects that can constitute limitations for participation are staff values and existing cultures in group homes (Bigby, \& Beadle-Brown, 2016 b; Clement \& Bigby, 2010) as well as structural barriers related to how the overall management and the organization support service managers in their everyday work (Beadle-Brown et al., 2014; Berlin Hallrup, 2012; Bigby, \& Beadle-Brown, 2016 b).

Social service for people with ID in Sweden is funded by taxes and regulated by the Act concerning support for persons with certain functional impairments (SFS 1993:387). Sweden has 290 politically driven and self-governing municipalities that are responsible for providing support in accordance with the Act. Service managers are responsible for implementing the intentions of the legislation on participation and ensuring that it is followed by all staff employed in group homes (The National Board of Health and Welfare, 2017). Moreover, the Act stipulates that participation is the key goal and describes that service managers in group homes should work closely with the staff in order to better support and guide them in daily work. Service manager's leadership in Sweden can thus be described in line with what Deveau 
\& McGill (2016) describe as frontline managers, which means coaching, counseling and supporting staff in daily service delivery.

The most common support for people with ID are day care activities and group homes (The National Board of Health and Welfare, 2018). There are approximately 27.000 people with ID residing in staffed group homes in Sweden and it is regulated that a maximum of six residents reside in each group home (The National Board of Health and Welfare, 2018). Concurrently, considering that the responsibility for providing support is decentralized to the municipalities it can be some differences between them.

Despite previous research, how service managers create opportunities for participation for adults with ID in Sweden as well as in other countries remains relatively unknown (BeadleBrown et al., 2014; Bigby, \& Beadle-Brown, 2016 a; Deveau \& McGill, 2016; Jackson 2011). Service managers, who are responsible for the day-to-day care of adults with ID in staffed group homes can be assumed to be in a position to improve staff performance which may increase opportunities for participation for residents in group homes. Therefore, the aim of the present study was to explore service managers' experiences of how the participation of adults with ID can be promoted in Swedish group homes.

\section{Method}

\section{Methodology and design}

This is a qualitative explorative study underpinned by a constructivist epistemological lens. The qualitative design was used to gain an understanding of service managers everyday experiences of how the participation of adults with ID can be promoted in Swedish group homes. The constructivist epistemological lens allowed an approach where the researchers and informants interacted and co-constructed knowledge based on their experiences. This implies that the researchers participated in the research process with the subjects in producing knowledge that 
is reflective to the informants reality (Lincoln, Lynham, \& Guba, 2018). Thus, the epistemological assumption is that participation for adults with ID and how that can be promoted by service managers is inherent in their experiences.

\section{Informants}

In this study, service managers are directly responsible for managing and supporting staff in group homes for adults with ID. Strategic sampling was employed to select 20 different municipalities representing large cities, small towns and rural areas. One service manager in each municipality was contacted by e-mail with information about the study and that a criterion for participation was at least one year's experience as a service manager of a group home for adults with ID. They were also informed that participation was voluntary and requested to reply to the e-mail within two months. Six informants declined participation, three due to lack of time while three gave no reason. Thus, 14 informants (13 women and one man) agreed to participate. Their work experience ranged from 4-35 years. Ethical approval for the study was granted by The Swedish Regional Ethical Review Board (Reg.No 2016-712).

\section{Setting}

The informants were service managers of group homes for adults over the age of 20, with ID who were autistic or had a condition resembling autism. They were all responsible for at least one group home with a maximum of six residents and some for up to five group homes, which meant approximately 6-35 residents and 20-60 part-time and permanent employees. While in some group homes the residents lived more or less independently, those included in the present study are staffed on a 24 hour basis. 


\section{Data Collection}

Individual interviews with 14 service managers was used to gain an understanding of their experiences of how the participation of adults with ID can be promoted in Swedish group homes. The time and place for each interview was agreed by the informants and the first author. The interviews were conducted between August and November 2016. Each interview started with the opening question "Can you please describe what participation for adults with ID means to you?”, followed by “How do you promote participation for adults with ID in a group home?”. Probing questions such as “What do you mean?” or "Can you tell me more about that?” were posed in order to facilitate deeper reflection on the part of the informants. The interviews lasted between 60 and 80 minutes, were performed in a secluded room, digitally recorded and transcribed verbatim by the first author.

\section{Data analysis}

The data analysis was conducted in accordance with thematic content analysis by Braun and Clarke (2006). Initially, the first author transcribed the recorded interviews, then all authors independently read the interviews several times. Each author identifyed and generated descriptive codes that grouped data based on similar experiences and topics. The next stage of analysis included all authors, coding and sorting the transcripts in order to organize them into patterns highlighting meaningful subthemes. Further, the analysis continued by identifying important themes collating all data extracts that had been coded. Once completed, the analysis provided repeated opportunities to cross-check raw data against emergent themes, thus ensuring credibility and trustworthy.Table 1 provides an example of the analysis process from data to codes to identify the main themes "Creating preconditions for participation” and " Barriers for promotion of participation” (Table 1).

Please, insert Table 1 here. 


\section{Findings}

The informants' experiences of how participation can be promoted in group homes for adults with ID consisted of two main themes; “Creating preconditions for participation” and "Barriers for promotion of participation”. Creating preconditions for participation comprised the following subthemes: Facilitating a trusting relationship and Supporting staff to increase participation. The second main theme; Barriers for promotion of participation included two subthemes: Restrictions that hinder participation and Managing while experiencing a lack of support (Figure 1). In the findings section, the term service user will be employed as a synonym for adults with ID.

Please, insert Figure 1 here.

\section{Creating preconditions for participation}

The informants described always putting the service users first and used various organizational structures and approaches to encourage staff to support and work in a way that increased service user participation. In order to find ways of creating preconditions for participation they created structures to facilitate a trusting relationship between staff and service users by ensuring that staff had certain responsibilities and enough time to get to know the service users. Another important aspect was using individualized implementation plans based on the needs of service users. To further fulfill the participation ideology, the informants provided supervision, used a reflected approach in day-to-day work with staff and applied strategies such as the participation model. 
TABLE 1: Development of codes and subthemes from data for the main themes "Creating preconditions for participation" and "Barriers for promotion of participation".

\begin{tabular}{llll}
\hline Quotes & Codes & Subthemes & Themes \\
\hline $\begin{array}{l}\text { "First, staff must create a relation- } \\
\text { ship built on trust...//" }\end{array}$ & Build trust & Facilitating a trusting relationship & $\begin{array}{l}\text { Creating preconditions } \\
\text { for participation }\end{array}$ \\
$\begin{array}{l}\text { "In addition to my support, I } \\
\text { arrange supervision...//" }\end{array}$ & Arrange supervision \\
$\begin{array}{l}\text { "Sometimes both staff and users } \\
\text { wishes are overruled } \\
\text { by next of kin...//" }\end{array}$ & Restrictions & Supporting staff to increase participation & $\begin{array}{l}\text { Creating preconditions } \\
\text { for participation }\end{array}$ \\
$\begin{array}{l}\text { "I miss the occupational } \\
\text { therapist...//" }\end{array}$ & Restrictions that hinder participation & $\begin{array}{l}\text { Barriers for promotion } \\
\text { of participation }\end{array}$ \\
\hline
\end{tabular}



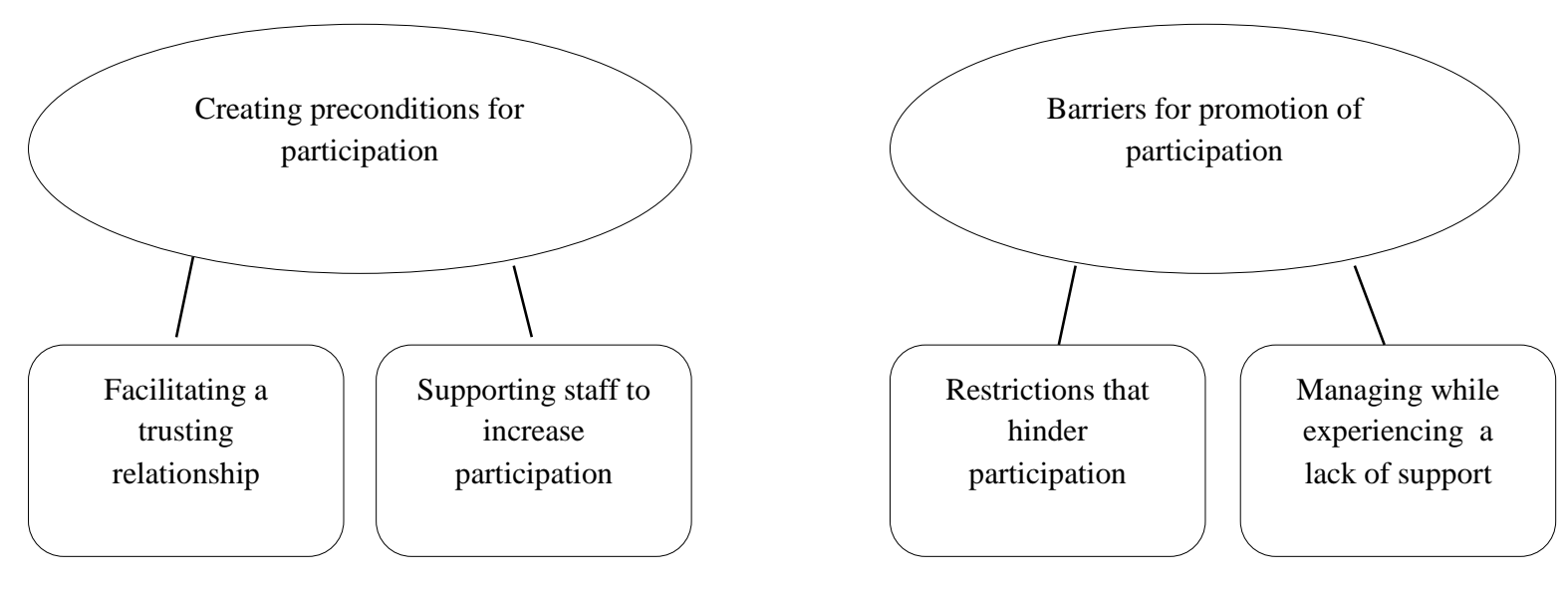

Figure 1 Thematic map of the two main themes and subthemes relating to service managers' experiences of how the participation of people with intellectual disabilities (ID) can be promoted in Swedish group homes 


\section{Facilitating a trusting relationship}

Facilitating participation meant that the informants assumed that staff initially needed to develop a trusting relationship with each service user. According to the informants, trust was crucial for service users' feelings of confidence. They believed that a trusting relationship was necessary and something that service users could benefit from when developing their own individual strategies for participating in everyday life. The following statement exemplifies this belief:

First, staff must create a relationship built on trust with the service users. It is important. If they have no relationship with the individual, nothing will work.

\section{(Informant 9)}

In order to facilitate a trusting relationship the informants provided their employees with sufficient time to get to know each service user. The informants explained that staff were given a certain responsibility in the group home, which meant that one staff member was a so-called “contact person” for a new resident. The contact-person had the responsibility for gaining a deeper understanding of the individual's needs and formulating an individually based “implementation plan”. This gave the service user an opportunity to be involved in how service and support were to be provided by staff and what such service and support should entail.

The implementation plan should be formulated without delay. When someone moves in here, she/he receives a contact person and then the formulation of the implementation plan starts immediately.

(Informant 1) 
The informants expressed that facilitating a trusting relationship was an ongoing process that was constantly developing as they tested new strategies and ways of working. This could involve the introduction of new activities to empower service users to further express their opinions.

We have taken it a step further...//...as we have a coordinator for participation, she ensures that service users receive assistance with communication tools so that they have a better opportunity to express themselves at meetings.

(Informant 10)

The informants also expressed that facilitating a trusting relationship to increase participation, meant encouraging and challenging service users to become even more active and visible, such as participating in the recruitement of new staff.

\section{Supporting staff to increase participation}

The informants also needed to support the staff in their everyday work by means of various strategies in order to successfully create preconditions for service user participation. One of the strategies for them was to be present in the group homes and assist staff members in organizing their daily work as means of increasing the focus on participation. Another important strategy for supporting staff in their efforts to increase participation was supervision. The informants sometimes provided one-to-one and group supervision themselves or in collaboration with professional supervisors.

In addition to my support, I arrange supervision for staff, which is necessary. For one group we have supervision regarding work place meetings and we also have 
individual supervision where the supervisor has been involved in the whole process.

(Informant 2)

The informants described having a reflective approach and challenging the staff members' assumptions and prejudices, such as when various ethical dilemmas arose, for example involving a service user with no verbal skills.

How can we find out what he wants? The staff came up with the idea of showing him pictures of various dishes and suddenly he pointed to the food he wanted or did not want. It is important to constantly challenge staff and give them the opportunity to reflect on what other options that are available for service users.

(Informant 13)

The informants also highlighted the importance of continuous staff education. They were convinced that the staff members needed in-depth knowledge and education about, for example, disabilities and pedagogical approaches.

It is important for the staff to have knowledge of disability legislation, but also about pedagogical approaches...in this municipality we have invested a lot in various forms of staff training.

(Informant 8)

The informants described the necessity of ensuring that staffing and schedules were based on when and how service users needed assistance and support. They also strived to stimulate that regular and recurring structures for participation was embedded in daily work. One such structure included to create a forum for dialouge such as the participation model between 
service users and a certain employee with responsibility as a supervisor. This model was considered as a necesseary strategy not only to adress the wishes and needs of service users, but also as a means to develop the staff's knowledge of the users' wishes and needs.

We call it the participation model, we have trained an employee with a certain responsibility who does not work in that specific group home who has meetings four times a year with service users. The model is based on the assumption that service users should express their preferences and opinions in relation to their accommodation in the group home.

(Informant 11)

\section{Barriers for promotion of participation}

Although the informants described opportunities for participation, they also expressed that from time to time they experienced barriers that prevented them from promoting increased participation. The barriers led to a tension between trying to enable participation in accordance with disability legislation and adapting to existing conditions and restrictions that limited their opportunity to do so. They described that there still exist restrictions that hinder participation such as attitudes by staff or next of kin and a lack of physical space in the group home. Informants also stated that managing was a sense of lacking necessary support by the overall management, due to financial or political decisions.

\section{Restrictions that hinder participation}

According to the informants, restrictions that hinder participation could be due to low staff turnover and the fact that employees had worked in the same group home for too long. The 
informants reported that staff members who had worked in the same place for many years found it more difficult to change their approach encouraging and promoting service user participation.

A few days ago I asked staff member in one of my group homes about what service users want and the answer I received was, What? What do you mean?

(Informant 6)

The attitudes of next of kin also contributed to restrictions in enabling participation, especially when they lacked knowledge about disability legislation and service users' right to influence and participate in daily life.

Next of kin have very strong opinions. Often the residents tell the staff what they would like, but their next of kin object. Sometimes both staff members' and service users’ wishes are overruled by next of kin.

(Informant 12)

According to the informants, there were also physical restrictions in the group homes, such as a lack of space enabling social interaction between service users, staff and visitors. Hence, service users had to stay in their apartments.

There is no real community room for service users, when you enter you go straight into the TV room. It is more like a giant hall or corridor. It is difficult for visitors and service users to meet socially, so instead service users stay in their own apartments.

(Informant 12) 
Managing while experiencing a lack of support

The informants were aware of the structural strategies, necessary for creating and increasing successful service user participation. At the same time, they felt limited by various aspects, such as the overall management, the organization or political directives that prioritized the need to adhere to the budget in the municipality. Such directives made the informants feel that they lacked necessary support from allied health professionals and instead they had to manage by themselves.

I miss the occupational therapist as that profession is important for making staff members understand at what level they should communicate and what communication service users might need.

(Informant 7)

The informants described that they sometimes felt lonely and lacked time, which led to a sense of resignation about developing the necessary improvements for staff.

I am alone and responsible for five group homes right now...//...the development ideas that I had, such as implementing the participation model, have grounded to a halt. I have no time to follow things up, it is not worth starting something new when I do not have enough time to make staff members familiar with it...or follow it up...right now it is sort of work done in vain.

(Informant 13)

Managing while experiencing a lack of support meant that the informants missed a clear vision on the part of the overall management which led to feelings of frustration, especially, when there was a high turnover among top-line and administrative managers. The informants described an occasional lack of understanding support from the overall management. The 
informants expressed that they felt overruled due to political decisions as well as governed by overarching systems, making them unable to influence and promote participation.

We are also governed by policies and union interests; for example, we must endeavor to ensure the right of all employees to work full-time...even if I do not need so many full-time employees. I prefer to focus instead on promoting and starting...such as the participation model for service users.

(Informant 1)

\section{Discussion}

Our study illustrates the significance service managers place on their responsibility for the promotion of service users opportunities for active participation. For this work, they describe the various strategies used to fulfill the intentions of the Swedish disability participation policy.

One such prominent strategy described in this study, was to be present as a leader providing support and instant feedback to the staff in their efforts to include service users' needs and perspectives. Thus, the informants regarded themselves as role models and interacted in dayto-day practice by observing and providing supervision to staff. This strategy indicates that daily support to staff is important in order to enable conditions for participation for service users in group homes. Based on these findings we argue that the service manager leadership described by the informants as being attentive to staff providing instant feedback and supervision may be compared to what earlier research define as practice leadership (Mansell \& Beadle-Brown 2012; Deveau \& McGill 2016). 
Another strategy stated by the informants was to establish a trusting relationship between staff and service users. The informants assumed that a trusting relationship was necessary to develop at an early stage in order to establish sustainable opportunities for participation. Similar findings have been reported by Collings et al., (2018) who highlight person-centred approaches, which describes that the relationship between practitioners and people with ID should be based on trust and to get to know the person before engaging them in further activities.

Furthermore, the participation model was put forward by yhe informants as a strategy that provided a structure for how participation for service users could be organized and as such it was considered an asset in achieving necessary quality goals. The model provided ways for the service managers how to create social and including arenas where service users and staff could meet. Our findings thereby contribute to previous research by Gullacksen and Hejdedal (2014) who state that the initial purpose of the participation model was to empower people with ID to be engaged in meaningful activities as well as in relationships with others. According to Gullacksen and Hejdedal (2014), the participation model can contribute to greater influence and participation as it allows an open space where service users can feel comfortable sharing their views in a group home. However, the model may also exclude people with severe and profound intellectual disabilities because they sometimes lack communication skills. Therefore, we suggest that future studies explore how the model can be developed to better suit the needs of this vulnerable group.

The informants described the occasional lack of support from overall management, which limited their efforts to promote participation and the implementation of the participation model. This can be seen as a serious shortcoming as a prerequisite for the participation model to lead to successful results is that the entire organization is willing to integrate the model (Gullacksen \& Hejdedal 2014). This suggests that it is important that the overall management ensure that service managers have enough resources to properly implement the participation model. 
Therefore, we suggest that the participation model is implemented and systematically tested in future studies.

The informant's experienced several barriers that limited their opportunity to enhance participation. Sometimes they felt abandoned as they lacked necessary support from other professionals such as occupational therapists due to financial directives. Further, service managers stated that staff who had worked for longer periods in group homes were less inclined to change their attitude to enable participation. In other words, it may mean that low staff turnover could contribute to a culture that is less likely to promote participation for service users. Similar findings were reported by Bigby (2012), that on the one hand, staff longevity may help to shape the culture of a group home, on the other hand it can perpetuate a culture where residents are regarded as “others”.

Findings in this study showed that service managers attempted to promote staff to deliver better disability practice based on the disability ideology of participation. Having the responsibility as a service manager and providing policies at a local level can be understood in the light of the concept of "street-level bureaucracy” described by Lipsky (1980). Lipsky (1980) argued that "decisions of street-level bureaucrats, the routines they establish and the devices they invent to cope with uncertainity and work pressures, effectively become the public policies they carry out” (p.xiii). In this study service managers can be described as street-level practioners trying to adapt and transform the national disability policy to professional values and local strategies. At the same time, a lack of support due to local political or financial decisions gave the service managers a sense of underperforming in their daily mission to promote participation. It may also imply that service managers struggle and manage alone while experiencing a lack of support for making good disability practice even better. 


\section{Strenghts and limitations}

The present study focused primarly on a strategic sample of fourteen service managers' experiences of how participation in daily life can be promoted for adults with intellectual disabilities in Swedish staffed group homes. Thus, a limitation of the study is that only a small number of service managers participated and the staff to service users ratio was not collected.

Several criterias were used to increase trustworthiness (Lincoln \& Guba 1985). Credibility involves giving the informants a voice through a description of their individual experiences. The emerging themes were discussed among the authors until consensus was achieved, thus ensuring credibility. Moreover, to enhance dependability, the first author conducted all the interviews using an open and inductive approach. Confirmability was strengthened by illustrating the themes using quotations from the informants. Taken toghether, this means that the findings may be transferable to similar contexts.

\section{Conclusions}

The findings contribute to an understanding of how service managers promote participation for adults with ID in Swedish staffed group homes. All service managers experienced that promoting participation in everyday life for service users was an important part of their mission and professional role. In parallell with how the service managers provided support for their staff, they also needed support themselves from various key actors within the organization in order to introduce structures aimed at increasing participation. Based on the outcome of this study, we suggest that structural strategies, such as coaching, supervision and reflection, to support staff are important but can be further developed. 


\section{Conflict of interest}

None.

\section{Acknowledgements}

The present authors would like to thank all the service managers who participated in this research project.

\section{Author contribution}

All authors contributed equally to this manuscript.

\section{Correspondence}




\section{References}

Bailey, M., Doody, O. \& Lyons, R. (2016). Surveying community nursing support for persons with an intellectual disability and palliative care needs. British Journal of Learning Disabilities, 44, 24-34. https://doi.org./10.1111/bld.12105

Beadle-Brown, J., Mansell, J., Ashman, B., Ockenden, J., Iles, R. \& Whelton, B. (2014). Practice leadership and active support in residential services for people with intellectual disabilities: an exploratory study. Journal of Intellectual Disability Research, 9, 838-850. https://doi.org/10.1111/jir.12099

Berlin Hallrup, L. (2012). The meaning of the lived experiences of adults with intellectual disabilities in a Swedish institutional care setting: a reflective lifeworld approach. Journal of Clinical Nursing, 23, 1583-1592. https://doi.org/10.1111/j.1365-2702.2012.04208

Bigby, C., Knox, M., Beadle-Brown, J., Clement, T. \& Mansell, J. (2012). Uncovering dimensions of informal culture in underperforming group homes for people with severe and profound intellectual disabilities. Intellectual and Developmental Disabilities, 50, 452-467. https://doi.org/10.1352/1934-9556-50.06.452

Bigby, C. (2012). Social inclusion and people with intellectual disability and challenging behaviour: A systematic review. Journal of Intellectual and Developmental Disability, 37, 360-374. https://doi.org/10.3109/13668250.2012.721878 
Bigby, C., Frawley, P. \& Ramcharan, P. (2014). Conceptualizing inclusive research with people with intellectual disability. Journal of Applied Research in Intellectual Disabilities, 27, 3-12. https://doi.org/10.1111/jar.12083

Bigby, C., Knox, M., Beadle-Brown, J. \& Clement T. (2015). “We just call them people”: Positive Regard as a dimension of culture in group homes for people with severe intellectual disability. Journal of Applied Research in Intellectual Disabilities, 28, 283-295. https://doi.org/10.1111/jar.12128

Bigby, C. \& Beadle-Brown, J. (2016 a). Culture in better group homes for people with severe and profound intellectual disabilities. Intellectual and Developmental Disabilities, 54, 316331. https://doi.org/10.1352/1934-9556-54.5.316

Bigby, C. \& Beadle-Brown, J. (2016 b). Improving quality of life outcomes in supported accommodation for people with intellectual disability: What makes a difference? Review. Journal of Applied Research in Intellectual Disabilities,1-19. https://doi.org/10.1111/jar12291

Bigby, C., Bould, E. \& Beadle-Brown, J. (2017). Implementation of active support over time in Australia. Journal of Intellectual \& Development Disability, 1-13. https://doi.org/10.3109/13668250.2017.13553681

Bigby, C., Andersson, S. \& Cameron, N. (2017). Identifying conceptualizations and theories of change embedded in interventions to facilitate community participation for people with intellectual disability: A scoping review. Journal of Applied Research in Intellectual Disabilities, 31, 165-180.https://doi.org/10.1111/jar.12390 
Braun, V. \& Clarke, V. (2006). Using thematic analysis in psychology.Qualitative Research in Psychology, 3, 77-101.https://doi.org/10.1191/1478088706qp063oa

Clement, T. \& Bigby, C. (2010). Group Homes for People with Intellectual Disabilities. Encouraging Inclusion and Participation. London, UK: Jessica Kingsley.

Collings, S., Dew, A. \& Dowse, L. (2018) Unpacking the complexity of planning with persons with cognitive disability and complex support needs. Journal of Applied Research in Intellectual Disabilities, 31, 142-151. https://doi.org/10.1111/jar.12381

Deveau, R. \& McGill, P. (2014). Practice leadership at the front line in supporting people with intellectual disabilities and challenging behaviour: A qualitative study of registered managers of community-based, staffed group homes. Journal of Applied Research in Intellectual Disabilities, 29, 266-277. https://doi.org/10.1111/jar.12178

Deveau, R. \& McGill, P. (2016). Leadership at the frontline: Impact of practice leadership management style on staff experience in services for people with intellectual disability and challenging behaviour. Journal of Intellectual and Developmental Disability, 39, 65-72. https://doi.org/10.3109/13668250.2013.865718

Gullacksen, A.C. \& Hejdedal, R.M. (2014). Delaktighetsmodellen - en väg mot empowerment. Uppföljningstudie av erfarenheter från Skåne (The model of participation-a path towards empowerment. Follow-up study of experiences from Skane). Lund, Sweden: FoU Välfärd Skåne. Skriftserie 2014:1. 
Jackson, R. (2011). Challenges of residential and community care: ” the times they are a changin”. Journal of Intellectual Disability Research, 55, 933-944.

https://doi.org/10.1111/j.1365-2788.2011.01461.x

Lincoln, Y. S. \& Guba, E.G. (1985). Naturalistic Inquiry. Newbury Park, California: Sage.

Lincoln, Y.S., Lynham, S.A., \& Guba, E.G. (2018). Paradigmatic Controversies, Contradictions, and Emerging Confluences, Revisted. In The SAGE Handbook of Qualitative Research, Fifth Edition. Editors, Denzin, N.K., \& Lincoln, Y.S. Thousand Oaks, California:Sage

Lipsky, M. (1980). Street Level Bureaucracy: Dilemmas of the individual in Public Services. Russell Sage Foundation, New York: Sage

Mansell, J. \& Beadle-Brown, J. (2012). Active Support: Enabling and Empowering People with Intellectual Disabilities. London: Jessica Kingsley.

SFS 1993:387. Lagen om stöd och service till vissa funktionshindrade (Act concerning support and service for persons with certain functional impairments). Stockholm, Sweden: Ministry of Health and Social Affairs.

The Swedish Agency for Participation (2015). Samlad Uppföljning Av Funktionshinderspolitiken HUR Är Läget? (Comprehensive Follow-up of Disability Policy How are things?). Stockholm, Sweden: The Swedish Agency for Participation. 
The National Board of Health and Welfare (2017). Vägar till ökad delaktighet. Kunskapstöd för socialtjänsten om arbete och stöd och service enligt LSS. (Roads for increased participation - Knowledge support for social services on work with support and service according to LSS) Stockholm, Sweden: The National Board of Health and Welfare.

The National Board of Health and Welfare (2018). Insatser och stöd till personer med funktionsnedsättning. Lägesrapport 2018. (Service and support for people with disabilities. Current status report) Stockholm, Sweden: The National Board of Health and Welfare.

United Nations (2006). Convention on the rights of persons with disabilities. New York: United Nations.

World Health Organization (2001). International Classification of Functioning, Disability and Health. Geneva, Switzerland: World Health Organization. 